\title{
Cognitive Radio: State of Research Domain in Next Generation Wireless Networks - A Critical Analysis
}

\author{
Jai Sukh Paul Singh \\ Dept. of Electronics Technology \\ Guru Nanak Dev University \\ Amritsar, India.
}

\author{
Jasvir Singh \\ Dept. of Electronics Technology \\ Guru Nanak Dev University \\ Amritsar, India.
}

\author{
A.S. Kang \\ Dept. of Electronics and Comm. \\ Panjab University \\ Chandigarh, India.
}

\begin{abstract}
Cognitive Radio (CR) is a comparatively new technology in which problems like underutilization of spectrum and spectrum scarcity is solved based on the revolutionary ideas. Cognitive Radio allows group of users to identify and access to available spectrum resources for their optimum use. Recent studies show that most of the assigned spectrum is underutilized. On the other hand, the increasing number of wireless multimedia applications leads to a spectrum scarcity. Cognitive Radio is proposed as a promising technology to solve the imbalance between spectrum scarcity and spectrum underutilization. In Cognitive Radio, spectrum sensing is done in order to locate the unused spectrum segments. This paper show the strength and capabilities of Cognitive Radio processes and what makes it more powerful over the other competitive radio. Main focus is given on Present State of Research, Architecture and Future Scope for CR. Stress is given on Application areas, where Cognitive Radio techniques can be implemented and proved out to have upper-hand than the available Intelligence and adapting Radios.
\end{abstract}

\section{Keywords}

Cognitive Radio, Software Defined Radio, Cognitive Cycle, Spectrum Sensing, Dynamic Spectrum Management, Spectrum Sharing, Spectrum Mobility, Primary User, Secondary User, Cognitive User.

\section{INTRODUCTION}

The aim of the Cognitive Radio technology is to provide the maximum efficiency of the spectrum to improve its utilization by using dynamic spectrum access techniques. The key to enable the maximum spectrum efficiency is to provide the capability to share the wireless channel with licensed users in the most efficient manner and this goal can be achieved by using spectrum management techniques that are dynamic and efficient. Cognitive radios are fully programmable wireless devices that can sense their environment and dynamically adapt their channel access methods, transmission waveform, spectrum utilization and networking protocols as needed for good network and application performance [2].The interesting feature of cognitive radio technology is one in which handsets would automatically use underutilized spectrum. Smartness of a radio is when it utilizes the available service from locally accessible wireless computer networks, and interacts with networks of preferred protocols, without any confusion in finding out the appropriate wireless network for audio video or data [3].Moreover, frequencies selection and utilization minimize/avoid interference with existing radio systems.

\subsection{Radios in Software Defined}

To exactly understand what basically Cognitive Radio is we have to start with Software Defined Radio (SDR), which is known to be the base platform for Cognitive Radio. Cognitive radios is a radio application that sit on top of an SDR, which as described before is implemented largely from General Purpose Processors (GPP's) and Digital Signal Processors (DSP's) [3]. Communication devices like the traditional radio system supports fixed number of channels and multiple frequencies but the problem is that channel and frequency must be chosen at the time of design and not at a later stage because there is no mechanism to bring about any post-design adjustment at the time of and as per actual requirement. In search of a better solution to this problem, a comparatively new concept called Software Defined Radio (SDR) was introduced. It was designed based on the idea of the traditional radio system but with a little change to its design. In SDR radio's physical layer behavior and functionalities are defined in software. In SDR different components of the radio communication system like amplifier and filters have been implemented in software instructions rather than the hardware [4].

\section{Software Defined Radio $=$ Traditional Radio + Software defined functionality}

Basically Software Defined Radio is defined [3] as "Radio in which entire or some of the physical layer functions are software defined". Software-Defined Radio (SDR) is a technology where software modules running on a generic hardware platform consisting of General Purpose Processors (GPP's) and Digital Signal Processor (DSP's) are used for the implementation of radio functions like Modulation at transmitter and Demodulation (detection/tuning) at receiver. Traditional radio's (hardware based radios) limits crossfunctionality and any modification can be done only using physical intervention which results in higher production costs. So, Software Defined Radio (SDR) as emerged out as an efficient and comparatively inexpensive solution to the above defined problem, using software upgrades. SDR can be used in implementation of applications like Bluetooth, Radar, GPRS, GPS, WLAN, Radar, WCDMA, etc. [3].

\subsection{Role of SDR in Wireless Communication}

Commercial Wireless network standards are continuously evolving from $2 \mathrm{G}$ to $2.5 \mathrm{G} / 3$ to $4 \mathrm{G}$ and now towards $5 \mathrm{G}$. This difference in networks of each generation, which significantly is the difference between Data-Link layer protocol standards causes problem to subscribes, wireless network operators and equipment manufacturers. With the coming of new generation subscribers are forced to update themselves to new handsets to take the advantage of upcoming services. Wireless network operators face problems with the problem of migration of the network from one generation to the next as majority of the subscriber's uses legacy handsets which are incompatible with newer generation network. So to overcome this network 
operators require costly equipment to migrate from one generation to next.

Moreover Air Interface and Data-Link layer protocols also differ across various geographies as GSM/TDMA are predominant in wireless networks over Europe while IS95/CDMA based wireless networks are predominant in countries like USA, which leads to the deployment of global roaming causing great inconvenience to subscribers who travel frequently between the continents [3]. To overcome this handset manufacturer had to face problems in building multimode handsets as accommodating such technology/facilities increases the cost and bulk of such handsets.SDR enables a family of products to be implemented using common platform architecture. With the arrival of new software, updated version of their respective product is made available and the updating the product, made the outdated product a latest product thereby reducing development cost. For Wireless Network Operators SDR adds latest features/capabilities to existing infrastructure. For subscribers SDR technology reduces costs to the subscriber by enabling them to communicate with whomever, whenever and in whatever manner they want. But as we know advantage and disadvantage are the two side of same coin, High power consumption, higher initial cost and higher processing power requirement are some of the drawbacks of SDR technology.

Although SDR brings a new concept and an important improvement of the idea of traditional radios, it is not completely fault-free. It does not have any intelligence and thus cannot take any decision of its own.

\subsection{Cognitive Radio}

To get over this limitation, a new concept called Cognitive Radio (CR) was improved on the basis of the idea of the SDR. Cognitive Radio was added with brain for intelligence of its own which imparted it a new capability of Decision making. Cognitive Radio provides a unique solution to the approach of spectrum underutilization problem. It can sense the surrounding environment and depending on the information and requirement of the situation, alters its physical layer and reconfigures itself. It can change the configuration so that it can handle complex situations. Moreover, it can adapt to the new situation [4].

\section{Cognitive Radio $=[$ Software Defined Radio $]+$ Intelligence + Reconfigurablility}

Cognitive Radio invented a new way of solving spectrum underutilization problem. It can sense the surrounding environment and try to find out the electromagnetic spectrum that is not utilized to its optimum capacity. After finding such range of frequencies, Cognitive Radio wants to utilize these properly by allowing the Secondary Users to utilize it in the vacuum slots. The main difference of Cognitive Radio from its predecessors is that it is defined by software and fully reconfigurable. All the logics in the Cognitive Radio can be implemented in software. Depending on the information Cognitive Radio can sense, change its behavior and needs which can be done through physical changes as well as by software instructions.

There are many definitions of CR and definitions are still being developed both in academic institutes and by various standards bodies. In a Layman term Cognitive Radio may be defined as [3] "A cognitive radio is a wireless communication system that intelligently utilizes any available side information about the Activity, Channel conditions,
Codebooks, Messages of other nodes with which it shares the spectrum."

Basically, cognitive radio could be defined as " $A$ radio that is cognitive", "Cogitate, ergo est." [5] ("Thinks", that makes the device "Cognitive" radio). Many researchers and public officials agree that upgrading a software radio's control processes will add significant value to software radio, but disagreement over the level of "cognition" required resulted in disagreement over the preciseness about the definition of cognitive radio. Some of the prominently definition about cognitive radio are discussed in detail.

In 1999 Joseph Mitola III coined the term "Cognitive Radio", and defined Cognitive Radio as [6] "A radio that employs model based reasoning to achieve a specified level of competence in radio related domains."

Later Simon Haykin defined Cognitive Radio as [7] "An intelligent wireless communication system which is aware of its surrounding environment (i.e., outside world), and uses the methodology of understanding by building to learn from the environment and adapt its internal states to statistical variations in the incoming $R F$ stimuli by making corresponding changes in certain operating parameters like Transmit-power, modulation strategy and carrier frequency in real-time, considering two primary objectives: $i$. Highly Reliable communications whenever and wherever needed. ii. Efficient utilization of the radio spectrum."

FCC focuses on operation of transmitter's defined cognitive radio as [8] " $A$ radio that can change its transmitter parameters based on interaction with the environment in which it operates."

NTIA [9], US Primary spectrum regulatory body defines cognitive radio as: "A radio or system that senses its operational electromagnetic environment and can autonomously and dynamically adjusts its radio operating parameters to modify system operation, such as mitigate interference, access secondary market, facilitate interoperability and maximize throughput"

The international spectrum regulatory community, ITU Wp8A, defines cognitive radio that focuses on capabilities as follows in [2] "A radio or system that senses and is aware of its operational environment and can dynamically and autonomously adjust its radio operating parameters accordingly."

IEEE USA defines CR as [10] "A radio frequency transmitter/receiver that is designed to intelligently detect whether a particular segment of the radio spectrum is currently in use, and to jump into and out of, as necessary the temporarily unused spectrum instantly, without any interference with the transmissions of other authorized users."

Similarly, SDR Forum [5] established two groups for cognitive radio and defined it as: "A radio that has, (1) awareness of changes in its environment and (2) in response to these changes adapts its operating characteristics in some way to improve its performance or to minimize a loss in performance."

However, the SDR Forum Special Interest Group [5] developing cognitive radio applications uses the following definition "An adaptive, multi dimensionally aware, autonomous radio system that learns from its experiences to plan, reason and decide future actions to meet the needs of the user." 
Virginia Tech Cognitive Radio Working Group defines Cognitive Radio as Adaptive Radio[11]:

"Adaptive radio are those which have following capabilities: -

i. Awareness about its environment and its capabilities

ii. Goal driven autonomous operation

iii. Learn/Understand how actions impact the goal

iv. Recall and correlate past actions, performance and environment."

The salient functionalities of all these above definitions, is summarized in Table 1. Some general capabilities among all of the definitions: -

Observation - directly or indirectly, system acquires information about its operating environment.

Adaptability - capable of changing its waveform.

Intelligence - capable of applying information to achieve target goal.

Combining these common features we can define Cognitive Radio as "Fully programmable wireless devices that can sense their environment and dynamically adapt their channel access method, transmission waveform, networking protocols and spectrum use needed for good network and application performance."

Table 1: Cognitive Radio Definition Matrix

\begin{tabular}{|c|c|c|c|c|c|c|c|c|c|c|c|}
\hline Definer & 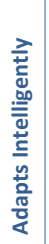 & 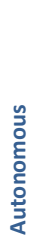 & 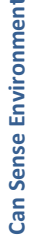 & 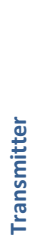 & 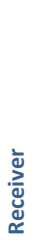 & 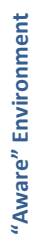 & 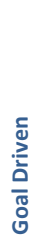 & 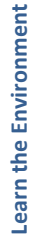 & 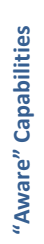 & 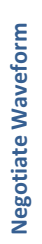 & 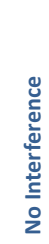 \\
\hline FCC [4] & $\checkmark$ & $\checkmark$ & $\checkmark$ & $\checkmark$ & & & & & & & \\
\hline Haykin [3] & $\checkmark$ & $\checkmark$ & $\checkmark$ & $\checkmark$ & $\checkmark$ & $\checkmark$ & $\checkmark$ & $\checkmark$ & & & \\
\hline IEEE USA [6] & $\checkmark$ & $\checkmark$ & $\checkmark$ & $\checkmark$ & $\checkmark$ & $\checkmark$ & & & & & $\checkmark$ \\
\hline ITU-R [10] & $\checkmark$ & $\checkmark$ & $\checkmark$ & $\checkmark$ & $\checkmark$ & $\checkmark$ & & & & & \\
\hline Mitola [2] & $\checkmark$ & $\checkmark$ & $\checkmark$ & $\checkmark$ & $\checkmark$ & $\checkmark$ & $\checkmark$ & $\checkmark$ & $\checkmark$ & $\checkmark$ & \\
\hline NTIA [5] & $\checkmark$ & $\checkmark$ & $\checkmark$ & $\checkmark$ & $\checkmark$ & $\checkmark$ & $\checkmark$ & & & & \\
\hline SDRF CRWG & $\checkmark$ & $\checkmark$ & $\checkmark$ & $\checkmark$ & $\checkmark$ & & $\checkmark$ & & & & \\
\hline SDRF SIG & $\checkmark$ & $\checkmark$ & $\checkmark$ & $\checkmark$ & $\checkmark$ & $\checkmark$ & $\checkmark$ & $\checkmark$ & $\checkmark$ & & \\
\hline VT CRWG[8] & $\checkmark$ & $\checkmark$ & $\checkmark$ & $\checkmark$ & $\checkmark$ & $\checkmark$ & $\checkmark$ & $\checkmark$ & $\checkmark$ & & \\
\hline
\end{tabular}

\section{PRESENT STATE OF RESEARCH ON COGNITIVE RADIO}

A driving feature of future network architectures are the mobile users, who want to access information resources while on the move, or whether travelling on a vehicle. Wireless technology is necessary to support the mobile user and adaptive and efficient use of radio spectrum is an important aspect of developing future network architectures [12]

Observing that in some locations or at some times of day, $70 \%$ of the allocated spectrum may be sitting idle, the FCC, in the Frequency allocation chart for United States shown in Figure 1 has recently recommended that significantly greater spectral efficiency could be realized by deploying wireless devices that can adjust themselves with the primary users, taking advantage of the available limited resources and minimizing interference. Thus, the discrepancy between spectrum allocation and spectrum use suggests that this spectrum shortage could be overcome by allowing more flexible spectrum utilization [13].

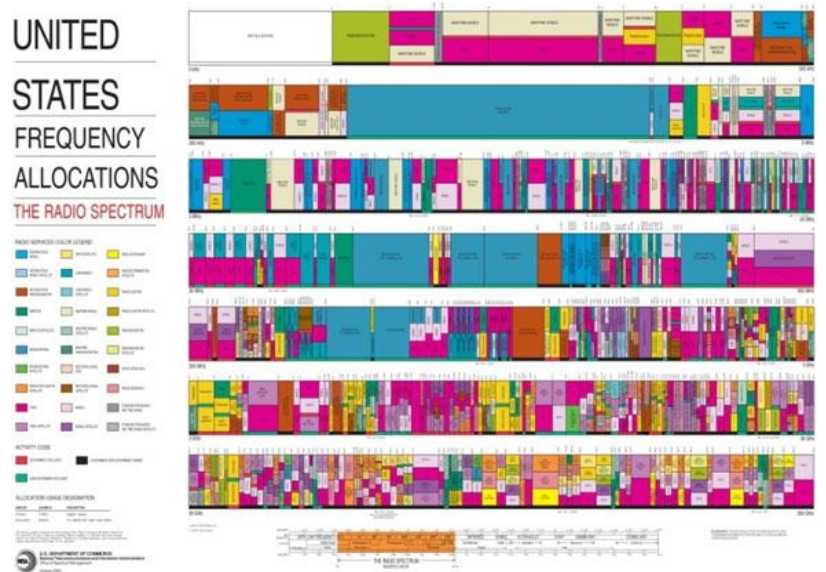

Figure 1: FCC Spectrum Allocation Chart [13].

Cognitive radios are aware of their surroundings and bandwidth availability and are able to tune dynamically with the spectrum usage based on geographical location, nearby radio devices and other factors. This feature of Cognitive Radio provides more efficient use of the spectrum as well as power consumption is reduced significantly. Moreover the feature of assigning priorities enables high priority communications to take precedence if required. Figure 2 shows use of Cognitive Radio in smart phones by Nokia Research Centre.

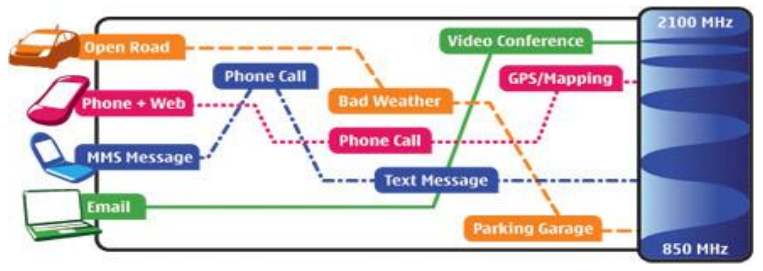

Figure 2: Cognitive Radio for Smart Phones [14].

Various devices will be able to detect other radios around them and work together to use more efficiently the limited spectrum band and allocate resources, which as a result will be more easy to communicate with their respective peer [14].

\section{STRENGTH OF COGNITIVE RADIO}

To optimize resource use, next generation networks require smart devices like Cognitive Radio to be able to model their location, networks, users and larger environment. Figure 3 show the various CR capabilities, which make it extraordinary from other radios. Based on monitored set of these parameters, Cognitive Radio can adapt to appropriate frequency bands, interfaces and protocols [6].

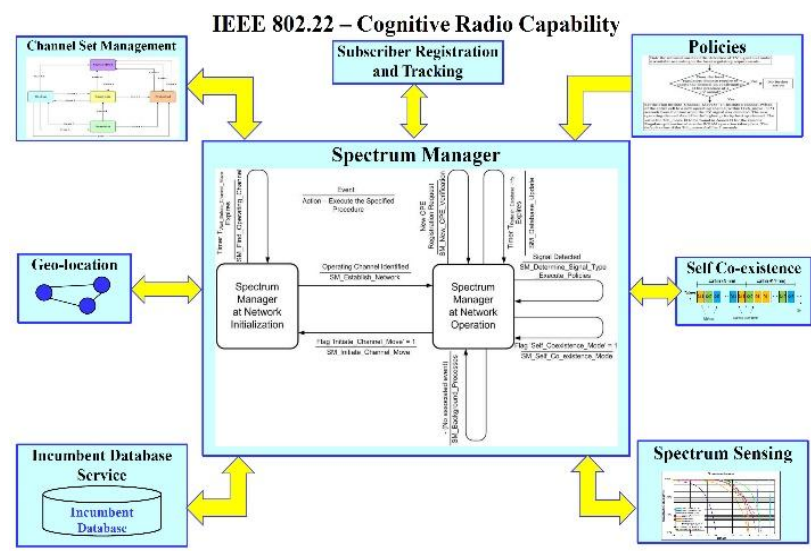

Figure 3: Cognitive Radio Capabilities [15]. 
Mitola [7] represented the major functions to adapt the transmission parameters in changing environments through a Cognitive Cycle. This six stage cognition cycle shown in Figure 4 is briefed as [20] follows: -

Observe: Get knowledge about the operating environment with the help of sensing and signaling mechanisms.

Orient: Evaluate the observed information for the determination of its significance and relevance.

Plan: Based on this evaluation, Cognitive Radio determines its options/alternatives for better resource optimization.

Decide: Take decision, taking in view the alternative that evaluates more favorably than other options (including the current on-going action.)

Act: Cognitive Radio implements the decisions taken for optimization of resources. Brought about changes are then reflected in the interference profile presented by the cognitive radio in the outside world as represent in Figure 4 below.

Learn: With the on-going process, Cognitive Radio utilizes its observations and its decisions to improve its future operation, creating new modeling states and alternatives and incorporating the feature of learning.

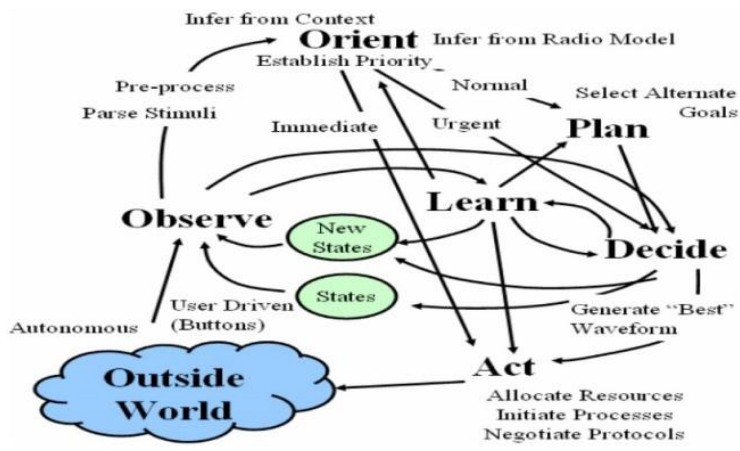

Figure 4: Basic Cognitive Cycle [1].

A CR can reliably sense wide bandwidth, detect the available empty spectrum band and use these empty slots for communication unless they are required by the PU. The air interface for Cognitive Radio is based on four main procedures [8]:-
i. Spectrum Sensing,
ii. Spectrum Management,
iii. Spectrum Sharing,
iv. Spectrum Mobility.

\subsection{Spectrum Sensing}

In context of Cognitive Radio, spectrum sensing refers to the detection of spectrum holes with the help of spectrum sensing techniques such as transmitter/energy detection, interference based detection, matched filters and cooperative detection [9]. Not only must the Cognitive Radio detect the spectrum holes, continuous monitoring of spectrum is also necessary. Time, accuracy and detection range are important considerations for sensing. Some associated problems in sensing are as follows:

False Alarm: Cognitive Radio detects the Primary User even if no Primary User is present. This is called False Alarm.

Missed Detection: Presence of Primary User in the surroundings of Cognitive Radio and it does not observe its presence, this is known as Missed Detection.

\subsection{Spectrum Management}

Spectrum management refers to acquiring the best available spectrum which includes spectrum analysis and then selecting the band according to user requirements. Various operating parameters and transmission parameters need be continuously analyzed so that the best combinations of parameters might be tuned to maintain the QoS. A number of optimization techniques have been used including artificial intelligence and soft computing techniques [6].

\subsection{Spectrum Sharing}

Once a Cognitive Radio knows its transmitting frequency, it informs its receiver about the band chosen so that a common communicating channel can be established. Besides, a fair spectrum scheduling method is to be provided, it can be regarded to be similar to generic MAC problems in existing systems [6].

\subsection{Spectrum Mobility}

Spectrum mobility or handover refers to the change of operating frequency or band. Mobility occurs when CR changes its frequency band upon detection of PU signal. To obtain high QoS, CR needs to switch to another frequency, maintaining seamless communication requirements during the transition to better spectrum. Data rate, throughput, SNR latency are some of the important parameters for deciding when handover is required for maintaining seamless connections [6].

\section{ARCHITECTURE AND LAYERS OF COGNITIVE RADIO}

In Nov 2004, the first worldwide effort was made to define a novel wireless air interface between MAC and PHY standard based on Cognitive Radios known as IEEE 802.22 Working Group (WG) [17]. As 802.22 is required to reuse the fallow TV spectrum without causing any harmful interference to Primary Users. IEEE 802.22 , mostly targeted at rural and remote areas having coverage range quite larger than 802.16 as depicted in Figure 5. Also, 802.16 do not include incumbent protection techniques necessary to operate in licensed bands [32]. IEEE 8022.22 has frequency range used in the VHF/UHF TV broadcast bands extends from 54 to 862 $\mathrm{MHz}$ depending on the various regulatory domains around the world [21].

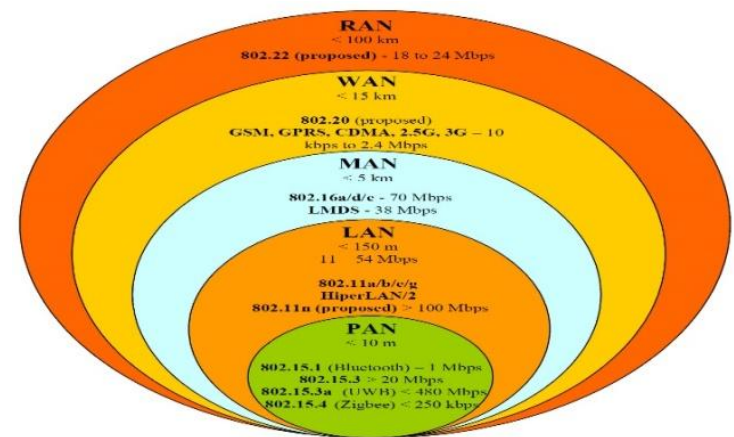

Figure 5: Comparison of 802.22 WRAN classifications with other popular wireless IEEE standards [32].

\subsection{Physical Layer}

Physical layer regulates interaction between data link layer and physical wireless medium [16]. Spectrum sensing is the main goal of the PHY layer of a cognitive radio. Cognitive radio accounts the situations where both primary and secondary users occupy the same channel space like in licensed band scenarios. It is also responsible for spectrum sensing and reconfiguration of the transmission parameters. Cognitive Radio can reconfigure its operating frequency, modulation, channel coding and output power without hardware replacement, this is the most significant difference between cognitive radio network and other wireless networks 
physical layer. Software defined radio (SDR) based RF frontend transmitters and receivers [19] are required for configurability of cognitive radio networks. Implementing RF front-end, heavy-weight signal processing algorithms, detecting weak signals, presence of PU while there are secondary users, are significant sensing problems in Cognitive Radio [20]. There is a close relation between the requirements and functionalities of dynamic spectrum management and communication techniques in Cognitive Radio Network as illustrated in Figure 6.

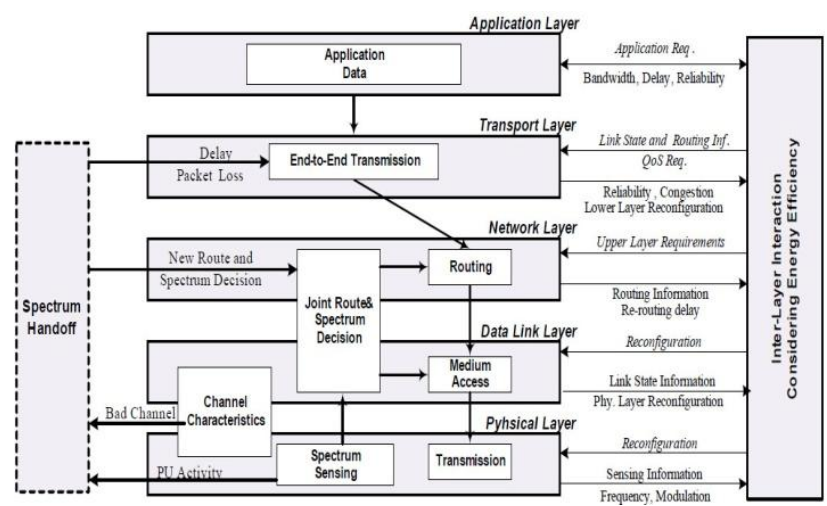

Figure 6: Interaction between the communication and dynamic spectrum management functionalities [16].

\subsubsection{Main Features of PHY Layer}

Following are the main features of Physical Layer [15] : -

PHY Transport: 802.22 use Orthogonal Frequency Division Multiplexing (OFDM) as transport mechanism.

Modulation: QPSK, 16-QAM and 64-QAM are supported. Coding: Convolutional Code is Mandatory. Turbo, LDPC or Shortened Block Turbo Codes are optional but recommended. Pilot Pattern: Each OFDM / OFDMA symbol is divided into sub-channels of 28 sub-carriers of which 4 are pilots, which are inserted every 7 sub-carriers. No frequency domain interpolation is required.

Net Spectral Efficiency: $0.624 \mathrm{bits} / \mathrm{s} / \mathrm{Hz}-3.12 \mathrm{bits} / \mathrm{s} / \mathrm{Hz}$. Spectral Mask: 802.22 have adopted the Spectral Mask requirements proposed by FCC.(200 tap FIR filter required)

To understand the architecture of IEEE 802.22 it is very important to understand, the core differences between 802.22(WRAN) and 802.16(WiMAX) [18] as they closely relate each other, both in their working and architecture. Table 2 tabulates the typical IEEE 802.22 features compared to IEEE 802.16, its closest "relative" among the IEEE 802 family [21].

\section{Table 2:IEEE 802.22 features compared to IEEE} 802.16[21].

\begin{tabular}{|l|l|l|}
\hline \multicolumn{1}{|c|}{ Features } & \multicolumn{1}{|c|}{ IEEE 802.22 } & \multicolumn{1}{c|}{ IEEE 802.16 } \\
\hline Air Interface & OFDMA & OFDMA, OFDM, Single \\
\hline $\begin{array}{l}\text { Fast Fourier } \\
\text { Transform }\end{array}$ & Single mode (2048) & $\begin{array}{l}\text { Multiple modes } \\
(2048,1024,512,128)\end{array}$ \\
\hline $\begin{array}{l}\text { OFDMA Channel } \\
\text { Profile (MHz) }\end{array}$ & $\begin{array}{l}\text { 6,7 or 8 (according to } \\
\text { regulatory domain) }\end{array}$ & $\begin{array}{l}28,20,17.5,14,10,7,3.5, \\
1.25\end{array}$ \\
\hline Burst Allocation & Linear & Two-dimensional \\
\hline $\begin{array}{l}\text { Sub-Carrier } \\
\text { Permutation }\end{array}$ & $\begin{array}{l}\text { Distributed with enhanced } \\
\text { Interleaver }\end{array}$ & Adjacent or Distributed \\
\hline $\begin{array}{l}\text { Multiple Antenna } \\
\text { Techniques }\end{array}$ & Not supported & $\begin{array}{l}\text { Support multiplexing, Space- } \\
\text { Time Coding and Beamforming }\end{array}$ \\
\hline $\begin{array}{l}\text { SuperFrame/Frame } \\
\text { Structure }\end{array}$ & $\begin{array}{l}\text { Support a super-frame } \\
\text { structure based on group of 16 } \\
\text { frames. Frame size: 10ms }\end{array}$ & $\begin{array}{l}\text { Super-frame is not } \\
\text { supported. Supported frame } \\
\text { size: } 2,5,10,20 m s\end{array}$ \\
\hline
\end{tabular}

\begin{tabular}{l|l|l|}
$\begin{array}{l}\text { Coexistence with } \\
\text { incumbents }\end{array}$ & $\begin{array}{l}\text { Spectrum sensing } \\
\text { management, geo-location } \\
\text { management, incumbent } \\
\text { database query and channel } \\
\text { management. }\end{array}$ & Not supported \\
\hline $\begin{array}{l}\text { Self-Coexistence } \\
\begin{array}{l}\text { Internetwork } \\
\text { Communications }\end{array}\end{array}$ & $\begin{array}{l}\text { Ovnamic spectrum sharing } \\
\text { beacon or over-the-IP-network }\end{array}$ & $\begin{array}{l}\text { Master frame assignment } \\
\text { (Primarily) }\end{array}$ \\
\hline
\end{tabular}

\subsection{Data Link Layer}

The primary function of CR's Data-Link layer is spectrum sharing, which can be seen in the Figure 7. Data Link layer is known for spectrum sharing because issues related to a radio's access to spectrum are typically concerns with MAC sublayer. Basic difference between generic MAC and MAC for cognitive radios is that coexistence between licensed and unlicensed users, dynamic selection of a frequency to transmit in a range of available spectrum and transmitter-receiver handshakes where two or more cognitive radios must agree on a mutual channel upon which to communicate [23]. Generally, efficient medium access control (MAC), and error control and correction are the main function of link layer.

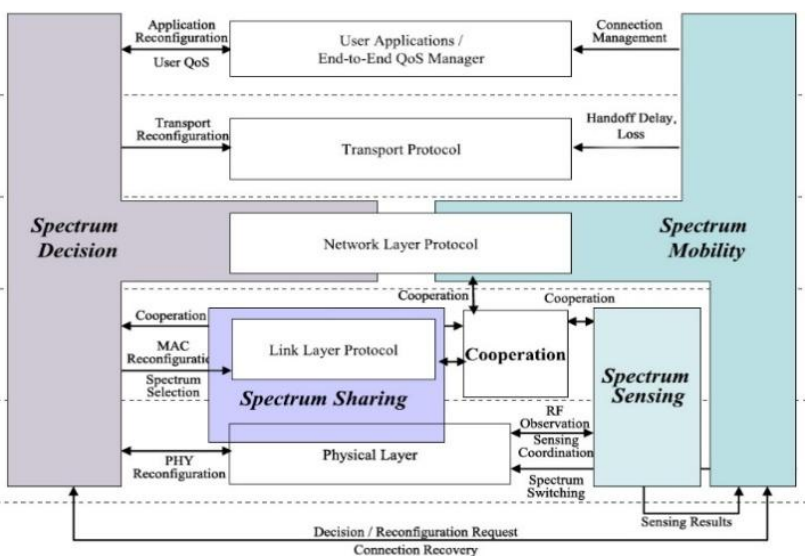

Figure 7: Simplified Cognitive Radio Layered structure as traditional OSI model [22].

Error Control: Forward Error Correction (FEC) and Automatic Repeat Request (ARQ) are the main error control schemes of wireless networks. But ARQ retransmission based mechanism causes extra energy consumption and reduces bandwidth utilization[16]. Therefore FEC schemes is a good alternative for resource-constrained cognitive radio network.

Medium Access Control: MAC protocol aims to provide network with means to access the medium in a fair and efficient manner, which is a challenging objective taking in view the limited resources, dense network deployment, and application-specific QoS requirements.

Table 3: Overview of MAC approaches developed for Cognitive Ad Hoc Networks [16].

\begin{tabular}{|l|l|l|l|}
\hline MAC Approach & $\begin{array}{l}\text { Disadvantages in } \\
\text { Cognitive Radio }\end{array}$ & $\begin{array}{l}\text { Reason to adopt } \\
\text { Cognitive Radio }\end{array}$ & \multicolumn{1}{|c|}{$\begin{array}{c}\text { Open Research } \\
\text { Issues }\end{array}$} \\
\hline $\begin{array}{l}\text { On-Demand } \\
\text { negotiation }\end{array}$ & $\begin{array}{l}\text { Contention due to } \\
\text { single channel for } \\
\text { all negotiations }\end{array}$ & $\begin{array}{l}\text { Suitable for bursty } \\
\text { traffic }\end{array}$ & $\begin{array}{l}\text { Coordination of } \\
\text { multiple control } \\
\text { channels required } \\
\text { for heavy traffic }\end{array}$ \\
\hline Home Channel & $\begin{array}{l}\text { Require multiple } \\
\text { transceiver }\end{array}$ & $\begin{array}{l}\text { Does not require } \\
\text { negotiation for each } \\
\text { packet (Reduces } \\
\text { power consumption) }\end{array}$ & $\begin{array}{l}\text { New approach } \\
\text { required for use with } \\
\text { single transceiver }\end{array}$ \\
\hline $\begin{array}{l}\text { Time-Division } \\
\text { based } \\
\text { negotiation }\end{array}$ & $\begin{array}{l}\text { Network-wide } \\
\text { synchronization is } \\
\text { required }\end{array}$ & Simple and few rules & $\begin{array}{l}\text { Get rid-off network- } \\
\text { wide } \\
\text { synchronization }\end{array}$ \\
\hline
\end{tabular}




\subsubsection{MAC Sub-Layer}

The IEEE 802.22 MAC provides mechanisms for flexible, supports cognitive capabilities and efficient data transmission for both reliable protection of incumbent services in the TV band and self-coexistence among 802.22 systems [21]. The IEEE 802.22 MAC is applicable to any region in the world and does not require country-specific parameter sets.

\subsubsection{Main Features of MAC}

Connection-oriented: This establishes connection, IDs and service flows which are dynamically created [15].

QoS: Various types of QoS services are supported shown in Table 4. ARQ supported, Uni-cast, Multi-cast and broadcast services are supported [15].

Table 4: QoS provided by IEEE 802.22 MAC Layer [15].

\begin{tabular}{|c|c|}
\hline QoS & Applications \\
\hline UGS & VolP, T1/E1 \\
\hline rtPS & MPEG Video Streaming \\
\hline nrtPS & FTP \\
\hline BE & E-mail \\
\hline Contention & BW request etc. \\
\hline
\end{tabular}

\section{Cognitive functionality [15]: -}

i. Dynamic and adaptive scheduling of quiet periods to allow the system to balance QoS requirements of users with the need to quiet down the network to support spectrum sensing. Quiet periods range from 1 symbol (approx. 1/3 ms) to one super-frame.

ii. Subscribers can alert the BS, the presence of user (incumbents) in a number of ways. Dedicated - Urgent Co-existence Situation (UCS) messages or low priority MAC messages.

iii. BS can ask one or more subscribers to move to another channel in a number of ways using Frame Control Header $(\mathrm{FCH})$ or dedicated MAC messages.

\subsubsection{LLC Sub-Layer}

LLC in 802.22 have no special difference for cognitive tasks.

\subsection{Network Layer}

Cognitive radio networks are not like traditional selforganizing wireless ad hoc networks. They are not designed to work with a single fixed frequency band. They can opportunistically utilize various spectral holes, white spaces, for peer-to-peer communications [16]. Cognitive network protocols supports variety of higher layer applications like voice, data, video, and mobile real-time services, as traditional wireless networks, moreover they have to aware of rapidly changing radio environment, access to multiple radio channels, and PHY and MAC dedicated spectrum usage. In Figure 7 creating stable CR Networks involve distribution and management PHY-Network layer and cross-layer information. Existing ad hoc cognitive radio routing schemes aim to provide joint spectrum and routing decisions, whereas routing schemes for other networks mainly aims to minimize energy consumption so do not handle dynamic spectrum access. However, predetermined routing is not suitable for dynamic topology caused by opportunistic channel access; so ondemand routing is advised for CR networks [24]-[27].

\subsection{Transport Layer}

Transport layer is mainly responsible for end-to-end reliable delivery of event readings and congestion control. With the detection of an event, sensor nodes inject high and busty traffic into the network, to achieve successful detection and tracking of an event signal, sufficient number of event readings must be reliably delivered to the receiver end and if the capacity of multi-hop network is exceeded at same time it would lead to congestion which wastes power and communication resources of network [16]. Clearly, there is a relation between reliability and energy-efficiency, which has been the main focus for proposed transport layer for Cognitive sensor networks. None of the available transport layer solutions for traditional wireless network, which grantees reliable delivery with minimum energy consumption and congestion avoidance can be considered for dynamic spectrum access, so there exists no transport layer solution for ad hoc cognitive radio networks either [28]-[31].

\subsection{Application Layer}

Application layer algorithms mainly deal with the generation of information and extracting the features of event signal being monitored to be communicated to the receiver end. Application layer also provide methods to query sensors, interest and data dissemination, data aggregation and fusion [23]. There is no application-layer protocol developed specifically for Cognitive Radio Network so far. Application layer protocol that analyses and organizes user queries in a heterogeneous network for efficient transmission is needed. Theoretically significant amount of research is done on data aggregation and fusion techniques for traditional radio networks [32]. Data aggregation and fusion techniques are used to increase estimation performance at the cost of communication and computational complexity. The justification in terms of energy consumption is that computation at a node consumes less energy than communication. As data aggregation and fusion reduce the number of transmissions, these techniques improves energy efficiency and network utilization. The nodes with better channel availability can send and receive more data in a more reliable manner, therefore these nodes can be assigned more tasks. Moreover priorities may be assigned to the nodes based on their tasks and spectrum availability to achieve fairness enabling nodes with less channel availability to access better channels [16].

\section{COGNITIVE RADIO APPLICATIONS}

With the upcoming of the technology for Cognitive Radio, many possibilities are being explored for using its functionalities and capabilities for various applications. There are many existing SDR techniques that can be enhanced with the use of cognitive radio features. Following are few frequently advocated applications of cognitive radio.

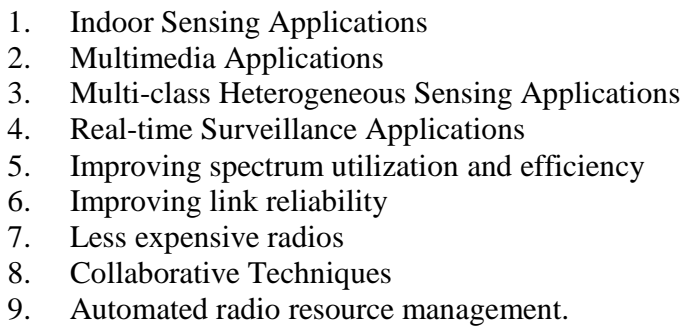

\subsection{Indoor Sensing Applications}

In Indoor applications like Tele-medicine, home monitoring, emergency networks, factory automation, generally require the existence of many small networks within a small area [34]. The main problem with indoor sensing applications is that the unlicensed bands, for indoor usage are often crowded [33]. So, conventional radio networks may experience significant challenges in achieving reliable communication due to packet losses, collisions and contention delays. To 
overcome this, using CR Network may help mitigate these challenges due to crowded spectrum.

\subsection{Multimedia Applications}

Reliable and on time delivery of event features in the form of multimedia, like, audio, still image, video, over traditional radio networks is an extremely challenging objective due to inherent high bandwidth demand of multimedia [35]. Unlike the traditional radio networks, Cognitive Radio networks can provide the freedom of dynamically changing communication channels according to the environmental conditions and application-specific Quality-Of-Service(QoS) requirements in term of bandwidth, bit error rates, and access delay [16]. Therefore, for multimedia communication Cognitive Radio networks may improve the performance of multimedia communication along the efficient overall spectrum utilization

\subsection{Multi-Class Heterogeneous Sensing Applications}

Some applications may require multiple radio networks with distinct sensing objectives to coexist over a common area [36]. Combination of all information gathered from these networks is fused to feed a single decision support. Similarly, in a single radio network, different channels may be deployed over the same area to sample the event signal over multiple dimensions, like, heat, humidity, location, motion, as well as audio visual readings of the target being monitored [16]. These heterogeneous networks introduce heterogeneity; therefore, using cognitive radio network, through the coordination and cooperative spectrum management, their individual performance as well as the overall spectrum utilization can be improved to a great extent.

\subsection{Real-Time Surveillance Applications}

Real-time surveillance applications like target detection and tracking require minimum channel access and communication delay. In traditional radio networks with fixed spectrum allocation, this objective cannot be always achieved, especially if the operating spectrum band is crowded [16]. Even, additional communication latency occurs traditional radio, if rerouting due to a link failure or degraded channel is caused. In Cognitive Radio Networks, it will access the available channel in such a way so that it can maintain minimum access and end-to-end communication delay for effective real-time surveillance applications. One typical realtime sensing application example is military surveillance applications which are highly delay-sensitive and also require high reliability. Moreover, with the spectrum handoff capability, tactical surveillance Cognitive Radio may be less susceptible to interception and jamming threats [16].

\subsection{Improving Spectrum Utilization and Efficiency}

With the increase in demand of Wireless technologies and wireless devices over past decade there is dramatic increase in the demand for electromagnetic spectrum but thanks to this approach to spectrum access, spectrum offer has not maintained with spectrum demand resulting in the looks of scarceness within the spectrum. But from the result of research performed by various agencies like FCC indicates that this assumption is away from reality as lot of spectrum is available as most of the spectrum allocated is still underutilized. Recent studies funded by National Science Foundation (NSF) for allocated spectrum utilization, at Kansas University found that average U.S. spectrum occupancy of $5.2 \%$ with a maximum occupancy of $13.2 \%$ in New York City.

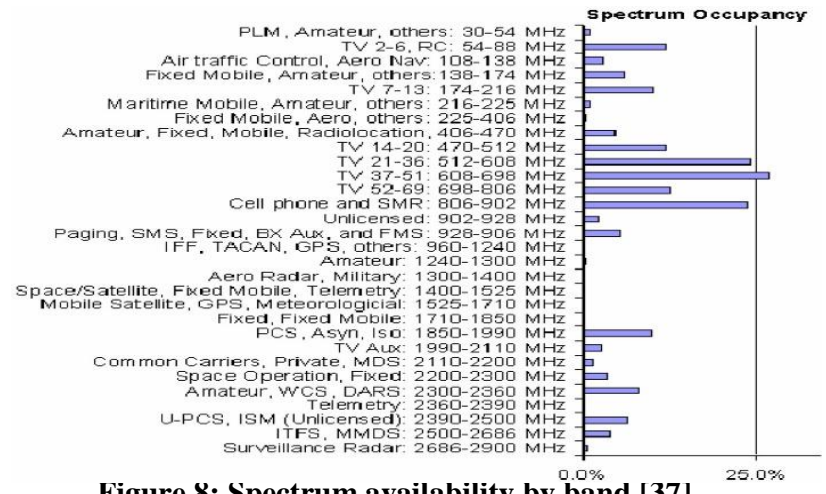

Figure 8: Spectrum availability by band [37].

Figure 8 show the specific measurements for following six locations: 1. River-bend Park, Great Falls, VA, 2. Tyson's Corner, VA, 3. National Science Foundation Roof, Arlington, VA, 4. SSC Roof, Vienna, VA 5. NRAO, Green-bank, WV, 6. New York City, NY, [37]. This comes to the point that dramatically increasing demand for spectrum has fostered a perception that spectrum is scarce but the fact is that spectrum is however poorly utilized.

\subsection{Improving Link Reliability}

After improving spectrum utilization, the next most important application of cognitive radio is improving link reliability. By adapting transmission power levels, error correction and using modulation can improve link reliability. However, a cognitive radio is a radio that is capable of remembering and learning from its past experiences and can go much beyond these simple adaptations. This is explained in an example as shown in Figure 9 shows the path that a mobile subscriber follow on his daily route within a city, and a significant drop of signal is shown in red is observed daily due to a coverage gap. After observing the drop at a particular point will be observed and cognitive radio would become aware of this situation. Learning from this Cognitive Radio could anticipate the coverage gap and alter the signal characteristics to overcome this problem.

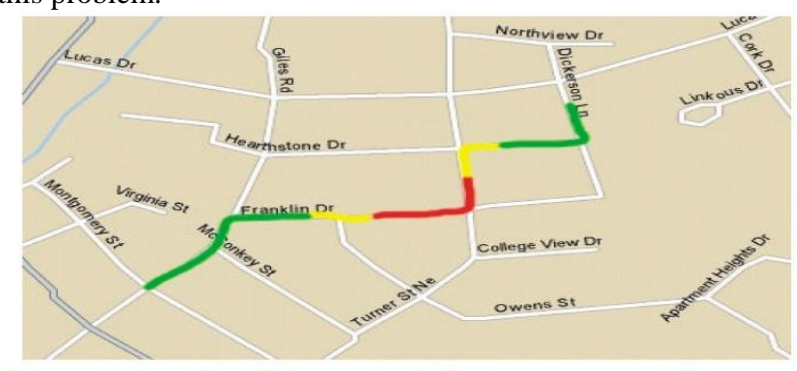

Signal Quality $\square$ Good $\square$ Transitional $\square$ Poor

Figure 9: Path and associated signal quality for Cognitive Radio [5].

\subsection{Less Expensive Radios}

By adding complexity to a radio's control processes will add up to the cost of the radio but this is not the case with Cognitive Radio. The cognitive control process significantly decreases the cost of the device when feature of Cognition is added to the Radio. So apparent paradox of adding features and low cost, it is important to note that many of the application based on Cognitive Radios may be represent as "Low Hanging Fruit" [5] which can be implemented via low complexity control processes. Moreover, implementing cognitive processes using software defined control process 
may in addition provide ease in computation especially when the target is to decrease the cost by improving the performance of analog components. Giving spectrum gain to accommodate lower performance analog components in place of improving capacity in a transmitter can result in signal energy outside of the intended band so including these low performance transmitter analog components can be implemented in Cognitive Radios or other "dumb" radios [5].

\subsection{Collaborative Techniques}

Collaborative Techniques are used in Collaborative Radio. So to understand collaborative techniques we should first know what Collaborative Radio is. A Radio that effectively utilizes the services of other radios achieves its goals or the goal of the network is known as Collaborative Radio [5]. Collaborative Radio could be implemented without fully implementing of cognitive radio but still Collaborative Radio can be viewed as an application of cognitive radio. As we all know that cognitive processes identify potential collaborators and intelligent observation processes thus facilitating the feature of distributed sensing which is known as the characteristic of many collaborative radio applications.

So to make Radio collaborate one of the most frequently ways is by implementing relay channels. In relay channel, a radio serves as an intermediate node between the client device and the access node. So this relaying process can be implemented at the relay node in two ways: -

i. Amplifying and forwarding the received signal in this case radio complexity is relatively low as the signal does not in actual received.

ii. Decoding and forwarding the signal. In this case radio complexity is much more as the relay has first to completely receive the signal and then forward.

However, the added complexity incurred by a decode-andforward approach is generally accompanied by improved performance (low latency waveforms being the most noticeable exception) so there exists a trade-off between the two approaches [5].

\subsection{Automated Radio Resource Management}

Practically when wireless network is setup, a wireless engineer spends few weeks tuning the radio parameters to get the best out of a network. Channels allocations between sectors, threshold for call-drops, times, power levels, antenna patterns and many other parameters are adjusted so as to improve performance of the network. With the increasing number of wireless networks the need to optimize wireless networks will become an increasingly important but will be impractical to be performed at home or in rapidly deployed networks. For instance, Virginia Tech spent months carefully planning and checking up on the deployment of its wireless LAN in order to maximize coverage with an acceptable capacity level - an unacceptable amount of time in a disaster response scenario. Because of its capacity to observe and learn how to improve its performance, cognitive radio networks could take over the task of post-deployment tuning and automatically update the radio parameters to best suit the needs of the particular deployment. Such an application would have a significant impact on rapidly deployed networks where emphasis, in home WLANs (which are rarely tuned), and in fixed commercial infrastructure where cognitive radio should be able to reduce the demand for post-deployment engineering.

\section{FUTURE SCOPE FOR COGNITIVE RADIO}

As Cognitive radio technology is an important innovation for the future of communications and likely to be a part of the new wireless standards, becoming almost a necessity for situations with large traffic and interoperability concerns. Moreover CR are devised to be used with telecommunications or computer network related disciplines but there are inadequate facilities to provide robustness and effective security. To overcome this, existing technologies will increase the complexity and new types of attack are possible. Therefore, innovative ideas are required to provide security to Cognitive Radio Networks and make them robust against crucial attacks, especially the attacks inherent to the Cognitive Radio functionality. So to make Cognitive Radio systems trustworthy, dependable and efficient, a comprehensive energy efficient mechanism is required to identify, remove or mitigate the attacks at any phases of the Cognitive Cycle.

\section{CONCLUSION}

Going through this paper, Cognitive Radio is proposed as a promising technology to solve the imbalance between spectrum scarcity and spectrum underutilization. Various CR capabilities like spectrum sensing, channel set-management, mobility, sharing, geo-location, self-co-existence subscriber registration and tracking and incumbent database makes it extra-ordinary from other radios and monitoring these set of parameters, Cognitive Radio can adapt to appropriate frequency bands, interfaces and protocols. Standard of Cognitive Radios is known as IEEE 802.22 Working Group can be beneficial in rural and remote areas as IEEE 802.22 has coverage range of about $100 \mathrm{~km}$. Due to the best overall performance, $\mathrm{CR}$ are expected to form a backbone for the next generation networks partially or wholly, which may to the great extent mitigate the spectrum scarcity problem. Moreover, during the emergency situations accessing the restricted unlicensed spectrum for transmission will be the best of the best application-able feature for Cognitive Radio.

\section{ACKNOWLEDGEMENT}

First author is highly thankful to Dr. Davinder Pal Sharma, DSP Research Lab, University of West Indies, Dr. B.P. Patil, Prof. \& Head, Army Institute of Technology, Pune and Dr. Suyeb Ahmed Khan, Head Dept. of Electronics and Comm. SBS College of Engineering and Technology, Patti for providing useful information as well as critical remarks. The valuable help rendered by Hardeep Singh, Research Fellow, DSP Research Lab, Department of Electronic Technology, GNDU Amritsar, India and Harjit Singh, Research Scholar, NIT-Jalandhar India are also acknowledged.

\section{REFERENCES}

[1] J. Mitola III, "Cognitive Radio: An Integrated Agent Architecture for Software Defined Radio", PhD Dissertation Royal Institute of Technology, Stockholm, Sweden, May, 2000.

[2] International spectrum regulatory community, ITU Wp8ATheInternational Telecommunication Union Available online: http://www.itu.int.

[3] "Software Defined Cognitive Radio using Matlab" Available online: http://www.scribd.com/doc/103610191/CognitiveRadio.

[4] J S Banerjee and K. Karmakar, "A Comparative Study on Cognitive Radio Implementation Issues", 
International Journal of Computer Applications (09758887), Vol. 45, No.15, May 2012.

[5] James O'Daniell Dissertation submitted to the Faculty of the Virginia Polytechnic Institute and State University, "Analysis and Design of Cognitive Radio Networks and Distributed Radio Resource Management Algorithms", September 6, 2006.

[6] J. Mitola, III, "Cognitive Radio for Flexible Multimedia Communications", Mobile Multimedia Communications, 1999. (MoMuC1999)1999 IEEE International Workshop on, pp. $3-10,1999$.

[7] S. Haykin, "Cognitive Radio: Brain-Empowered Wireless Communications", IEEE Journal on Selected Areas in Communications, Vol. 23, No.2, Feb. 2005.

[8] Federal Communications Commission, "FCC- ET Docket No. 03-108", March 11, 2005.

[9] National Telecommunications and Information Administration on FCC ET Docket No. 03-108, "Facilitating Opportunities for Flexible, Efficient, and Reliable Spectrum Use Employing Cognitive Radio Technologies", February 15, 2005.

[10] "Improving Spectrum Usage through Cognitive Radio Technology", IEEE USA Position, Nov 13, 2003, Available online:http://www.ieeeusa.org/policy/positions/cognitiveradio.asp

[11] "Cognitive Radio Definition", Virginia Tech Cognitive Radio Work Group Wiki. Available online: http://support.mprg.org/dokuwiki/doku.php?id=cognitive_radio:-definition

[12] Amna Saad Kamil and Ibrahim Khider, "Open Research issues in Cognitive Radio".

[13] Federal Communications Commission, Cognitive Radio Technologies Proceeding.

[14] Cognitive Radio for Smart Phones Available online: $\mathrm{http} / / /$ research.nokia.com/cognitive_radio

[15] Apurva N. Mody and Gerald Chouinard, "IEEE 802.22 Wireless Regional Area Networks Enabling Rural Broadband Wireless Access Using Cognitive Radio Technology", doc.: IEEE 802.22-10/0073r03, June 2010.

[16] Ozgur B. Akan, Osman B. Karli and Ozgur Ergul, "Cognitive Radio Sensor Networks".

[17] IEEE 802.22 Working Group on Wireless Regional Area Networks, http://www.ieee802.org/22/.

[18] IEEE 802.16 Working Group on Broadband Wireless Access, http://www.ieee802.org/16/.

[19] V. Blaschke, S. Nagel and F. K. Jondral, "Mechanisms for the Adaptation of the Physical Layer in a Cognitive Radio", in Proc. 9th European Conference on Wireless Technology, pp. 41-46, Sept. 2006.

[20] D. Cabric and R. W. Brodersen, "Physical Layer Design Issues Unique to Cognitive Radio Systems", in Proc. IEEE PIMRC 2005, Vol.2, pp. 759-763, Sept. 2005.

[21] Carl R. Stevenson, et al. "IEEE 802.22: The First Cognitive Radio Wireless Regional Area Network Standard", IEEE STANDARDS IN COMMUNICATIONS AND NETWORKING IEEE Communications Magazine, January 2009.

[22] Lance Hester and Ahmad D. Ridley, "Cognitive Radio Networks: Not Your Father's Wireless Network", A
NOBLIS Publication, The Telecommunications Review pp. 44-54, 2008

[23] I. F. Akyildiz, W. Y. Lee, M. C. Vuran, and S. Mohanty, "Next Generation/ Dynamic Spectrum Access/Cognitive Radio Wireless Networks: A Survey", Computer Networks, Vol. 50, No.13, pp. 2127-2159, 2006.

[24] S. Krishnamurthy, M. Thoppian, S. Venkatesan and R. Prakash, "Control Channel based MAC Layer Configuration, Routing and Situation Awareness for Cognitive Radio Networks", in Proc. IEEE MILCOM 2005, Oct. 2005.

[25] Jamal N. Al-Karaki and Ahmed E. Kamal, "Routing Techniques in Wireless Sensor Networks: a Survey", IEEE Wireless Communications, Vol. 11, No.6, pp. 6-28, Dec. 2004.

[26] G. Cheng, W. Liu, Y. Li and W. Cheng , "Spectrum Aware On-Demand Routing in Cognitive Radio Networks", in Proc. DySPAN 2007, pp. 571-574, Apr. 2007.

[27] C. Xin, "A Novel Layered Graph Model for Topology Formation and Routing in Dynamic Spectrum Access Networks", in Proc. IEEE DySPAN 2005, pp. 308-317, Nov. 2005

[28] C. Wang, M. Daneshmand, B. Li and K. Sohraby, "A survey of Transport protocols for Wireless Sensor Networks", IEEE Network, pp. 34-40, Vol. 20, No.3, 2006.

[29] S.J. Park, R. Vedantham, R. Sivakumar, and I. F. Akyildiz, "A scalable approach for reliable downstream data delivery in wireless sensor networks", in Proc. ACM MOBIHOC 2004, pp. 78-89, May 2004.

[30] O. B. Akan and I. F. Akyildiz, "Event-to-sink reliable transport in wireless sensor networks", IEEE/ACM Trans. Networking, Vol. 13, No.5, pp. 1003-1016, Oct. 2005.

[31] C. Wan and S. B. Eisenman, "CODA: Congestion Detection and Avoidance in Sensor Networks", in Proc. ACM SenSys 2003, pp. 266-279, Nov. 2003.

[32] Carlos Cordeiro, Kiran Challapali, and Dagnachew Birru, "IEEE 802.22: An Introduction to the First Wireless Standard based on Cognitive Radios", Journal of Communications, Vol. 1, No.1, pp. 38-47, April 2006.

[33] G. Zhou, J. A. Stankovic and S. H. Son, "Crowded Spectrum in Wireless Sensor Networks", in Proc. Third Workshop on Embedded Networked Sensors, 2006.

[34] S. Byun, I. Balasingham and X. Liang, "Dynamic Spectrum Allocation in Wireless Cognitive Sensor Networks: Improving Fairness and Energy Efficiency", in Proc. IEEE VTC 2008, pp. 1-5, Sep. 2008.

[35] E. Gurses and O. B. Akan, "Multimedia Communication in Wireless Sensor Networks", Annals of Telecommunications, Vol. 60, No. 7-8, pp. 799-827, July-August 2005.

[36] A. K. M. Azad and J. Kamruzzaman, “A Framework for Collaborative Multi Class Heterogeneous Wireless Sensor Networks”, Proc. IEEE ICC 2008, pp. 4396-4401, May 2008.

[37] M. McHenry, "NSF Spectrum Occupancy Measurements Project Summary", Aug. 15, 2005. Available online: http://www.sharedspectrum.com/?section=nsf_measurem ents. 